Copyright ( 2019 University of Bucharest Printed in Romania. All rights reserved

ISSN print: $1224-5984$

ISSN online: $2248-3942$
Rom Biotechnol Lett. 2019; 24(5): 851-855

doi: $10.25083 / \mathrm{rbl} / 24.5 / 851.855$

Received for publication, January, 3, 2018

Accepted, October, 18, 2018

\title{
Review
}

\section{Considerations Regarding GCMAF Treatement in Breast Cancer}

\author{
DAN NICOLAE PĂDURARU ${ }^{1,2}$, ALEXANDRA BOUARIU ${ }^{2}$, DANIEL \\ ION $^{1,2}$, OCTAVIAN ANDRONIC ${ }^{2}$, MIHAI CRISTIAN DUMITRAȘCU ${ }^{1,2}$, \\ ALEXANDRA BOLOCAN ${ }^{1,2}$
}

\author{
${ }^{1}$ University of Medicine and Pharmacy "Carol Davila", 37 Dionisie Lupu Str., 020021, \\ Bucharest, Romania \\ ${ }^{2}$ Bucharest University Hospital, 169 Splaiul Independenței, 050098, Bucharest, Romania \\ Authors' contributions: all authors have contributed in equal quantities
}

\begin{abstract}
Breast cancer is known as one of the most commonly occurring cancer which can be diagnosed in women. The number of new cases of breast cancer in female patients, both in early stages and in advanced stages, has slowly, but continuously increased in the last decades. Immunotherapy has been used in recent years in the treatment of many types of cancer, due to its reduced adverse reactions and a considerable tolerance when administrated compared to currently used therapies such as chemotherapy, radiation and surgery (1). GcMAF is a protein-derived macrophage-activating factor, a new and unapproved medication that has shown promising results in treating breast cancer (2). This review aims to summarize the studies and case reports presenting the GcMAF experience in breast cancer therapy.
\end{abstract}

Keywords Breast cancer therapy, GcMAF, immunotherapy.

To cite this article: PĂDURARU DN, BOUARIU A, ION D, ANDRONIC O, DUMITRAȘCU MC, BOLOCAN A. Considerations Regarding GCMAF Treatement in Breast Cancer. Rom Biotechnol Lett. 2019; 24(5): 851-855. DOI: 10.25083/rb1/24.5/851.855 


\section{Introduction}

Cancer, in general, is known to be a major and important causes of mortality and morbidity in the human population. Breast cancer is the most commonly occurring cancer in female patients (3). The number of new cases of breast cancer in female patients, both in early and in advanced stages, has, in the last decades slowly, but continuously increased (4). Studies that followed the incidence of breast cancer in women, have reported that the recently diagnosed cases of patients with breast cancer occur in $40 \%$ of cases in women older than 65 years of age and the majority of these women have an advanced stage of the neoplastic disease (5). Although breast cancer therapy has been constantly developed in the last decades, the mortality rate in patients with breast cancer has continued to rise (5), (6).

Immunotherapy has been used in recent years in the treatment of many types of cancer, due to its reduced adverse reactions and a considerable tolerance when administrated, compared to currently used therapies such as chemotherapy, radiation and surgery (1). Biological-based medication was designed to interfere with various mechanisms that were found in cancerous cells and tissues and promote the development and the resistance against the normally defense responses of the human body (2). Some of the purposes of these biologically-based drugs are to interfere with the angiogenesis mechanisms, with tumour genes that inhibit the normal process of changed cells removal in the human body and with various proteins found on the cell surface which send different messages inside the cell (6). Breast cancer responds to conventional therapies (including chemotherapy, radiation, hormonal therapy) especially in early stages, while the metastatic tumours are less sensitive to this type of treatment. Thus, immunotherapy can be a valuable option with less adverse effects in hormone refractory and chemoresistant breast cancer (5).

The research in cancer immunotherapy focused over the years on the role of different peptide and cells in breast cancer and the possibilities of becoming a target in cancer treatment (7), (2). T-cells, dendritic cells, natural killer cells and macrophages are major participants in the body's response to cancer and therefore cancer immunotherapy aims to mediate the activity of these cells (2), (7). Bevacizumab, a new antibody known to inhibit the vascular endothelial growth factor (VEGF) and erlotinib, an inhibitor that blocks the epidermal growth factor receptor (EGFR), are two available chemotherapy methods, but the results in the improvement of the survival rates are poor (2), (8). The explanation of such a modest improvement of the survival rates is that the source of the neoplastic cells stands, although these treatments are made to inhibit the tumour growth and spreading (8), (2). Deficiencies in the defense mechanism of macrophages and neutrophils are observed using the microscopic examination of cancerous tissue (9), (2). A new theory considers that neutrophils and macrophages can be used to remove the origin of cancerous cells and therefore they can be a promising new alternative in the treatment of breast cancer (9), (2). GcMAF is a the protein derived from the macrophage-activating factor, an unapproved medication studied for the future possibility to be used in the treatment of breast cancer.

In a recent study, was administrated inside the cancerous cells an amount of BCG (Bacille Calmette Guerin), or other bacterial cells and shortly after, as a result, they induced inflammation of cancerous tissues. Via inflammation both local tumours, as well as metastasized cells can regress, suggesting the development of specific immunity mechanisms against the tumours (10), (11). Inflammation in noncancerous tissues has not shown the same significant effect in comparation to the cancerous tissues (10), (11). Normal tissues release during inflammation a special type of lipid metabolites known as lyso-phosphatidylcholine and other lysophospholipids and these substances activate the macrophages and the body general defense machanisms (12), (13). Another type of lipid metabolites, at least 400 times more potent to activate macrophages, known as lysoalkylphospohlipids and alkylglycerols, are released during the inflammation process in cancerous cells. (14), (11). Highly activated macrophages are considered to have a tumoricidal effect, and this result explains why inflammation in cancerous tissues could become a potential mechanism to interfere in the treatment of breast cancer (11); practically, highly activated macrophages not only destroy cancerous cells, but also can remove them from the body (14), (15), (2).

\section{Material and Methods}

The research was conducted using PubMed, Scopus and WebofScience research platforms and used search formula: GcMAF and breast cancer or breast neoplasia. The research focused on the period 2000-2018 and included only English articles.

\section{Results}

Playing an important role in phagocytosis, macrophages are cells that internalize and destroy pathogens, releasing then different types of substances named cytokins. Macrophages can infiltrate tumours and are found at most tumour sites (1), (16). Therefore, macrophages have critical roles in antitumour immunity (1), (16).

One new described serum protein, with the average molecular weight of $55 \mathrm{kDa}$, a vitamin D-binding protein or Gc globulin, named the Gc protein has been seen to have a significant role in cancerous cells. The Gc protein is made by the liver and the chemical structure is similar to other proteins from the albumin superfamily (9). The Gc protein seems to have various roles in normal tissues, such as carrying and depositing vitamin $\mathrm{D}$, eliminating the extracellular G-actin and increasing the chemotactic activity of the component $\mathrm{C} 5 \mathrm{a}$ for neutrophils when inflammation occures (9), (16), (17). A glucose-modified protein called Gc protein-derived macrophage activating factor (GcMAF) has been observed to activate the 
macrophage (9), (17). Gc globulin plays a significant role in the immune system regulation (17), osteoclastic activity (18), in the primary response of the body to foreign unknown particles such as the immunodeficiency virus, in sepsis (19) and in antitumoural mechanisms (16). A Gc globulin modified protein is capable to affect the activation of immune cells increasing the anticancer activity (2), (17).

In some cancerous cell lines, the GcMAF precursor activity it is shown to disappear or to be decreased (2). This is the the consequence of the deglycosylated alpha $\mathrm{N}$-acetylgalactosaminidase (nagalase) secreted by the cancerous cells (20). An increased amount of nagalase has been found in patients with cancer, viral infections with HIV and Influenza virus, while healthy individuals secrete minimal levels of nagalase with no significant effect (21). Nagalase inhibits macrophage activation and generates severe immunosuppression in advanced cancer patients (13). It is observed that the quantity of nagalase has a reciprocal relation with the GcMAF level and the suppression of the immune system (2). Therefore studies show that in some types of cancer, the measurable quantity of nagalase in serum can play a prognostic role (2), (13), (22). The presence of nagalase in serum and its quantity, correlates with tumour weight and it has been shown that as GcMAF therapy continues, the activity of the protein called nagalase reduces (2), (13).

Another theory that suggests the use of GcMAF in cancer immunotherapy comes from its effects in angiogenesis inhibition (23). Angiogenesis is the process which can determine further development of cancerous cells and many agents used in chemotherapy or immunotheraphy methods have been considered for their role in angiogenesis inhibition (2). GcMAF can stop angiogenesis caused by pro-inflammatory prostaglandin E1 (2), (24). GcMAF stimulates the formation in mononuclear cells of peripheral blood of the 3-5-cyclic adenosine monophosphate (cAMP), which through the CD36 receptor blocks the initiation of angiogenesis (2), (24). As an important statement for future studies, the tumoricidal effect of macrophages via GcMAF and the contribution in chemotaxis of the cytokins released in the process, are the most important mechanisms towards angiogenesis (2).

\section{GcMAF and Breast Cancer Therapy}

Yamamoto et al showed, in 2008, in a study (11) which included 16 nonanemic females with breast cancer in advanced metastatic stages, the health improvement after the administration of GcMAF (11). In spite of detecting a high quantity of Nagalase in patients' serums, the use of GcMAF is a valuable procedure for cancer therapy (11). It was seen that the use of GcMAF determines an important activation of the macrophages not only to healthy humans, but also to patients with advanced metastatic cancer, validating the theory that said that serum nagalase doesn't increase the effect of GcMAF (11). In this study was observed that due to the time of 6 days required for a quantity of the activated macrophages to reduce to half its initial value (25), GcMAF was administered only once a week, via an intramuscular injection. GcMAF has rapidly spread systemically, including the cancerous tissues. The results were interesting: first, it was observed that the tumor associated macrophages were not activated by GcMAF; second, the phagocytosis and the superoxide capacities of all activated macrophages increased, independent of the stage of cancer (11). The macrophages activated by GcMAF recognize not only cancerous cells, but also can internalize and eliminate various bacteria (Escherichia coli, Salmonella typhi, Pseudomonas aeruginosa, Bacillus subtilis, Clostridium perfrigens, Mycobacterium tuberculosis, viruses (rubella, herpes simplex, parainflueza and influeza virus, HIV type 1) (26). The study shows that undifferentiated tumour cells, such as adenocarcinoma cells founded in breast cancer and other types of cancer such as prostate cancer, are detected and eradicated particularly than squamous carcinoma cells, with well-differentiated cancer cells (11).

The same study shows that during GcMAF therapy, in the majority of the breast cancer patients the serum Nagalase level decreased rapidly from the beginning of the administration and than followed a period in which the level slowly decreased (11). Therefore, the research of the cancer cell-specific Nagalase and the GcMAF treatment in advanced metastatic breast cancer can lead to another new biological-treatment possibility. The Nagalase level can be specifically followed during GcMAF therapy (23), (11), (25). Approximately $85 \%$ of breast cancer cells (MDA-MB-231 and MCF7) were identified and annihilated by the macrophages activated by GcMAF, in a different period of time, according to the presence or absence of serum pacient (or IgG fraction), respectively in 4 hours, or in 18 hours. These results suggest that the activated macrophages discover and destroy the cancer cells preferentially via Fc-receptor and that GcMAF-activated macrophages enhance a large quantity of Fc-receptors (11). The macrophage activation is an important mechanism in cancer immunotherapy as we understand from the present study, but in order to be statistically significant, it needs to be confirmed by other studies.

Pacini et al (3) decided to observe, in vitro, in 2012, the outcome of the commercial GcMAF, available on the research market, on a large human breast cancer cell line known as MCF-7. The study follows the GcMAF effect on cell growth, structure, vimentin expression and angiogenesis in neoplastic tissues (3). The results showed that after the incubation of MCF-7 human breast cancer cell with GcMAF in a dose-dependent manner, the rapid growth was significantly inhibited in comparation with the incubation of MCF-7 human breast cancer cell alone when the rapid growth appeared after $72 \mathrm{~h}$. The differences in the cell homogenity, structure and size were also evident and the inhibition of angiogenesis by GcMAF goes independent of other experimental pro-angiogenic factors, used frequently on experimental models. These results mentain and confirm the hypothesis that GcMAF is a molecule with multiple properties against the neoplastic disease (3).

In 2013, Thyer L et al (27) researched the tumoricidal effect of GcMAF activated macrophages on breast 
neoplastic cells, independent of the presence of other types of factors (27). It was observed that in the absence of GcMAF, macrophages promote none effect on human breast neoplastic cells and their irregular structure appearance was maintained (27). When GcMAF (100 ng/mL) was added to human breast neoplastic cells, the same type of macrophages were specifically activated and in 72 hours after incubation, the human breast cancer cells changed significant in structure, activating the mechanisms of apoptosis on the breast neoplastic cancerous tissues (27). The research showed that the macrophages activated by GcMAF initiate the mechanism of apoptosis in human breast cancer cell and as a result, not only the local cancerous cells, but also the spread ones in other parts of the body significantly shrink (27).

Inui $T$ et al presented in 2014 a case report of a 55year-old female, diagnosed with breast hormone receptor positive cancer (1). From the beginning, she was treated just by lumpectomy and no other additional treatment. After 4 years she presented with metastatic breast cancer with important symptoms and she was administrated second-generation high-dose GcMAF. In less than 4 months the patients symptoms decreased and her axillary tumour changed in size and disappeared completely using a combination of sonodynamic therapy, GcMAF and hormone therapy (1). This case report suggests that GcMAF can be used together with other approved specific therapies, such as sonodynamic therapy and hormone treatment, showing a better outcome for patients with neoplastic disease, with reduced adverse reactions and a positive immunological tolerance (28).

\section{Discussions and Conclusions}

The specific biological actions of GcMAF were first observed in 1994 (16). Shortly afterward GcMAF antitumoural properties were described in trial with BALB/c mice bearing Ehrlich tumour with ascites(28).

Gc-MAF has been studied for use not only in brest cancer, but also in other types of cancer, such as thyroid (29), lung (30), liver (31), pancreatic (31), bladder and ovarian cancer and tongue squamous carcinoma (32). Another particular aspect is that GcMAF has also been studied in relation with noncancerous diseases such as autism (33), multiple sclerosis (34), chronic fatigue syndrome (CFS) (35), juvenile osteoporosis (36) and systemic lupus erythematosus (37).

The existent evidence indicate that the machanisms against cancer induced by GcMAF use, include the tumoricidal macrophages specific effect, the inhibition of the new tumor-induced angiogenesis and through this the inhibition of cancer cell growth and the spread potential.

In conclusion, GcMAF shows great potential as a cancer immunotherapy alternative and it is required to perform further studies in order to observe the efficiency of this protein as a peculiar cancer immunotherapy option on a large group of patients.

\section{Aknowledgements}

Ethics approval and consent to participate: Not applicable.

Consent for publication: Not applicable. database.

Availability of data and material: Open data on each

Competing interests: no competing interests.

Funding: Not applicable.

Acknowledgements: no acknowledgements.

\section{Bibliography}

1. INUI T, MAKITA K, MIURA H, et al. Case report: A breast cancer patient treated with GcMAF, Sonodynamic Therapy and Hormone Therapy. Anticancer Research. 214; 34(4589-94).

2. SABURI E, SABURI A, GHANEI M. Gc-MAF promising role for cancer immunotherapy: From bench to bedside approach. Caspasia J Intern Med. 2017; $8(4)(228-238)$.

3. NITIPIR, C; NICULAE, D; ORLOV, C; BARBU, MA; POPESCU, B; POPA, AM; PANTEA, AMS; STANCIU, AE; GALATEANU, B; GINGHINA, O; PAPADAKIS, GZ; IZOTOV, BN; SPANDIDOS, DA; TSATSAKIS, AM; NEGREI, C. Update on radionuclide therapy in oncology,ONCOLOGY

4. SILLIMAN R, BALDUCCI L, GOODWIN J. Breast cancer care in old age; what we know, don $\mathrm{t}$ know and do. J Natl Cancer Inst. 1993; 85(190-199).

5. CHEON S, AGARWAL A, POPOVIC M, et al. The accuracy of clinicians predictions of survival in advanced cancer: a review. Ann Palliat Med. 2016; 5(22-9).

6. KIRKWOOD J, BUTTERFIELD L, TARHINI A, et al. Immunotherapy of cancer in 2012. CA Cancer J Clin. 2012; 62(309-35).

7. JOHNSON B, KABBINAVAR F, FEHRENBACHER L, et al. ATLAS: randomized, double-blind, placebocontroled, phase IIIB trial comparing bevacizumab therapy with o without erlotinib, after completion of chemotherapy, with bavacizumab for first-line treatment of advanced non-small-cell lung cancer. J Clin Oncol. 2013; 31(3926-34).

8. DICKLER M, RUGO H, EBERLE C, et al. A phase II trial of erlotinib in combination with bevacizumab in pstients with metastatic breast cancer. Clin Cancer Res. 2008; 14(7878-83).

9. NAGASAWA H, UTO Y, SASAKI H, et al. Gc protein (vitamin D-binding protein): Gc genotyping and Gc MAF precursor activity. Anticancer Res. 2005; 25(3689-95).

10. ZBAR B, TANAKA T. Immunotherapy of cancer: regression of tumors after intralesional injection of living Mycobacterium bovis. Science. 1971; 172 (271-3).

11. YAMAMOTO N, SUYAMA H, et al. Immunotherapy of metastatic breast cancer patients with vitamin 
D-binding protein-derived macrophage activating factor (GcMAF). Int J Cancer. 2008; 122(461-7).

12. YAMAMOTO N, NGWENYA B. Activation of macrophages by lysophospholipids, and ether derivatives of neutral lipids and phospholipids. Cancer Res. 1987; 47(2008-13).

13. YAMAMOTO N, SUYAMA H, YAMAMOTO N. Immunotherapy for Prostate Cancer with Gc ProteinDerived Macrophage-Activating Factor, GcMAF. Translational Oncology. 2008; 1(2)(65-72).

14. YAMAMOTO N, HOMMA $S$, et al. Activation of mouse macrophages by alkylglycerols, inflammation products of cancerous tissues. Cancer Res. 1988; 48(6044-9).

15. KLOSTERGAARD J. Macrophage tumoricidal mechanisms. Res Immunol. 1993; 144(274-6).

16. YAMAMOTO N, HOMMA S. Vitamin D3 binding protein (group-specific component) is a precursor for the macrophage-activating signal factor from lysophosphatidylcholine-treated lymphocytes. Proc Natl Acad Sci USA. 1991; 88(8539-43).

17. SPEECKAERT M, HUANG G, DELANGHE J, et al. Biological and clinical aspects of the vitamin D binding protein (Gc globulin) ant its polymorphism. Clin Chim Acta. 2006; 372(33-42).

18. SWAMY N, GHOSH S, SCHNEIDER G, et al. Baculovirus-expressed vitamin D-binding protein-macrophage activating factor (DBP-maf) activates osteoclasts and binding of 25-hydroxyvitamin $\mathrm{D}(3)$ does not influence this activity. J Cell Biochem. 2001; 81(535-46).

19. YAMAMOTO N, USHIJIMA N, KOGA Y. Immunotherapy of HIV-infected patients with Gc protein-derived macrophage activating factor (GcMAF). J Med Virol. 2009; 81(1)(16-26).

20. MOHAMAD S, NAGASAWA H, UTO Y, et al. Tumor cell alpha $\mathrm{N}$ acetygalatosaminidase activity and its involvement in GcMAF related macrophage activation. Comp Biochem Physiol A Mol Integr Physiol. 2002; 132(1-8).

21. REDDI A, SANKARAYANAN K, ARULAJ H, et al. Serum alpha $\mathrm{N}$ acetylgalctosaminidase is associated with diagnosis/prognosis of patients with squamous cell carcinoma of the uterine cervix. Cancer Lett. 2000; 158(61-4).

22. REHDER D, NELSON R, BORGES C. Glycosylation status of vitamin D binding protein in cancer patients. Protein Sci. 2009; 18(2036-42).

23. Allard B, TURCOTTE M, SPRING K, et al. Anti CD37 therapy impairs tumor angiogenesis. Int $\mathrm{J}$ Cancer. 2014; 134(1466-73).

24. PACCINI S, MORUCCI G, PUNZI T, et al. Effect of paricalcitol and GcMAF on angiogenesis and human peripheral blood mononuclear cell proliferation and signaling. J Nephrol. 2012; 24(77-81).

25. YAMAMOTO N. Preparation of potent macrophage activating factors derived from cloned vitamin D binding protein and its domain and their therapeutic usage for cancer, HIV-infection and osteopetrosis. US Patient. 2002; 6410(269).
26. YAMAMOTO N, UEDA M. Eradication of HIV by treatment of HIV infected/AIDS patients with vitamin D-binding protein (Gc protein) derived macrophage activating factor (GcMAF). Proc Int Cong Imm. 2004; (197-200).

27. CIOCIRLAN, M; DRAGHIA, L; MANUC, D; PANTEA-STOIAN, A; CAZAN, AR; HUSARSBURLAN, I; DUTEI, CA; CIOCIRLAN, M; MONICA, PC; DICULESCU, M; MANUC, M. NUTRITIONAL STATUS OF PATIENTS WITH DIGESTIVE CANCERS, INTERDIAB 2017: DIABETES MELLITUS IN INTERNAL MEDICINE, Book Series: International Conference on Interdisciplinary Management of Diabetes Mellitus and its Complications, Pages: 132-138, Published: 2017, Proceedings Paper Conference, Conference: $3^{\text {rd }}$ International Conference on Interdisciplinary Management of Diabetes Mellitus and its Complications (INTERDIAB), Bucharest, ROMANIA, MAR 02-04, 2017

28. YAMAMOTO N, NARAPARAJU V. Immunotherapy of BALB/c mice bearing Ehrlich ascites tumor with vitamin $\mathrm{D}$-binding protein-derived macrophage activating factor. Cancer Res. 1997; 57(2187-2193).

29. CHAIYASIT K, TOSHIO I, WIWANITKIT V. The use of Gc protein-derived macrophage activating factor for management of thyroid cancer. J Cancer Res Ther. 2015; 11(1041).

30. INUI T, AMITANI H, KUBO K, et al. Case report: a non-small cell lung cancer patient treated with GcMAF, sonodynamic therapy and tumor treating fields. Anticancer Res. 2016; 36(3767-70).

31. INUI T, KUCHIIKE D, KUBO K, et al. Clinical experience of integrative cancer immunotherapy with GcMAF. Anticancer Res. 2013; 33(2917-9).

32. THYER L, WARD E, SMITH R, et al. GC proteinderived macrophage-activating factor decreases alphaacetylgalactosaminidase levels in advanced cancer patients. Oncoimmunology. 2013; 2(257-69).

33. BRADSTREET J, VOGELAAR E, THYER L. Initial observations of elevated alpha-N-acetylgalactosaminidase activity associated with autism and observed reductions from Gc protein--macrophage activating factor injections. Autism Insights. 2012; 4(31).

34. INUI T, KATSUURA G, KUBO K, et al. Case report: GcMAF treatment in a patient with multiple sclerosis. Anticancer Res. 2016; 36(3771-4).

35. INUI T, KUBO K, KUCHIIKE D, et al. Oral colostrum macrophage-activating factor for serious infection and chronic fatigue syndrome: three case reports. Anticancer Res. 2015; 35(4545-9).

36. YAMAMOTO N, NARAPARAJU V, ORCHARD P. Defective lymphocyte glycosidases in the macrophage activation cascade of juvenile osteopetrosis. Blood. 1996; 88(1473-8).

37. YAMAMOTO N, NARAPARAJU V, MOORE M, et al. Deglycosylation of serum vitamin D3-binding protein by alpha-N-acetylgalactosaminidase detected in the plasma of patients with systemic lupus erythematosus. Clin Immunol Immunopathol. 1997; 82(290-8). 